\title{
Thick beryllium coatings by magnetron sputtering
}

H. Wu, A. Nikroo, K. Youngblood, K. Moreno, D. Wu, T. Fuller, C. Alford, J. Hayes, A. Detor, M. Wong, A. Hamza, T. van Buuren, E. Chason

June 13, 2011

2011 MRS Spring Meeting and Exhibit San Francisco, CA, United States April 25, 2011 through April 29, 2011 
This document was prepared as an account of work sponsored by an agency of the United States government. Neither the United States government nor Lawrence Livermore National Security, LLC, nor any of their employees makes any warranty, expressed or implied, or assumes any legal liability or responsibility for the accuracy, completeness, or usefulness of any information, apparatus, product, or process disclosed, or represents that its use would not infringe privately owned rights. Reference herein to any specific commercial product, process, or service by trade name, trademark, manufacturer, or otherwise does not necessarily constitute or imply its endorsement, recommendation, or favoring by the United States government or Lawrence Livermore National Security, LLC. The views and opinions of authors expressed herein do not necessarily state or reflect those of the United States government or Lawrence Livermore National Security, LLC, and shall not be used for advertising or product endorsement purposes. 


\title{
Thick Beryllium Coatings by Magnetron Sputtering
}

\author{
H. Xu, ${ }^{1}$ C. Alford, ${ }^{2}$ Eric Chason, ${ }^{3}$ A. Detor, ${ }^{2}$ T. Fuller, ${ }^{1}$ A. Hamza, ${ }^{2}$ J. Hayes, ${ }^{1}$ K.A. Moreno, ${ }^{1}$ \\ A. Nikroo, ${ }^{1}$ T. van Buuren, ${ }^{2}$ M. Wang, ${ }^{2}$ D. Wu, and K.P. Youngblood ${ }^{1}$ \\ ${ }^{1}$ General Atomics, P.O. Box 85608, San Diego, CA 92186-5608, USA \\ ${ }^{2}$ Lawrence Livermore National Laboratory, Materials Science and Technology Division, 7000 \\ East Avenue, Livermore, CA 94550, USA \\ ${ }^{3}$ Brown University, Department of Engineering, Providence, RI, USA
}

\begin{abstract}
Thick $(>150 \mu \mathrm{m})$ beryllium coatings are studied as an ablator material of interest for fusion fuel capsules for the National Ignition Facility (NIF). As an added complication, the coatings are deposited on mm-scale spherical substrates, as opposed to flats. DC magnetron sputtering is used because of the relative controllability of the processing temperature and energy of the deposits. We used ultra small angle x-ray spectroscopy (USAXS) to characterize the void fraction and distribution along the spherical surface. We investigated the void structure using a combination focused ion beam (FIB) and scanning electron microscope (SEM), along with transmission electron microscopy (TEM). Our results show a few volume percent of voids and a typical void diameter of less than two hundred nanometers. Understanding how the stresses in the deposited material develop with thickness is important so that we can minimize film cracking and delamination. To that end, an in-situ multiple optical beam stress sensor (MOSS) was used to measure the stress behavior of thick Beryllium coatings on flat substrates as the material was being deposited. We will show how the film stress saturates with thickness and changes with pressure.
\end{abstract}

\section{INTRODUCTION}

Beryllium is one of the ablator materials for National Ignition Facility (NIF) due to its low x-ray opacity and higher density. In particular, a graded copper-doped Be capsule is desired for ignition experiments. The capsule requires thick Be coatings (up to $160 \mu \mathrm{m}$ ) which are made on spherical mandrels, typically $\mathrm{CH}$ in composition [1]. The coating has to be carried out at low temperatures to avoid any deformation of the mandrel in order to produce uniform wall thickness and smooth and spherical surface finish. The coating must be dense with low void fraction and be leak-free when filled with $\mathrm{D}_{2}$ fuel gas. DC Magnetron sputtering is used to produce the coating because of the energetics of the depositing species and relative low coating temperatures [2].

The coating process has been described previously in several articles [2-5]. Typically, Be coatings on a spherical surface show a consistent columnar microstructure with acceptable void content and size. However, the Be coating suffers from sufficient interconnected porosity leading 
to rapid gas leakage. The measurements of $\mathrm{D}_{2}$ leak half-life at low temperature and ambient temperature indicated the leak is molecular flow through nanochannels [5]. Ion-assisted physical vapor deposition (IPVD) is the most commonly used method to produce dense films and to coat high aspect ratio trenches [6-10]. Using IPVD for growth of thin films has led to improved microstructures, improved adhesion, and improved coverage of three-dimensional substrates. Therefore, IPVD is a suitable approach to achieve high-density coating on spherical substrates used for making NIF capsules. IPVD techniques similar to those described in the literature has been employed here to produce Be coatings of NIF capsules and improved coating microstructure has been achieved.

Deposited films under stress can result in deformation, cracking and delamination. Because of the requirements of sphericity and surface roughness of Be coating on spherical substrates for NIF targets, it is essential to understand the Be film stress, especially the evolution of the stress during coating thick Be films.

\section{EXPERIMENTAL PROCEDURES}

Beryllium coatings were prepared in a vacuum chamber pumped by a cryopump. The base pressure was typically low $10^{-6}$ to middle $10^{-7}$ Torr. For coating on spherical mandrels, a bounce pan is used so the spherical substrates can be agitated during the coating for better uniformity. Be coating was produced by magnetron sputtering of Be targets onto $\sim 2 \mathrm{~mm}$ diameter glow discharge polymer spheres (mainly $\mathrm{CH}$ ) in 5-10 mTorr Ar environment. The distance between substrate and magnetron is a few $\mathrm{cm}$, which is not close enough to cause overheating of the substrate. To enhance the plasma density near substrates and supply extra flux of energized ion to the growing films, a secondary plasma was introduced near the substrate region and a negative biasing was added to the substrate to extract ions. The details of this ion-assisted physical vapor deposition (IPVD) process have been described elsewhere [11].

Be coatings were fractured to examine the cross section by scanning electron microscope (SEM). The detailed microstructures were analyzed by focus ion beam (FIB) lift and transmission electron microscopy (TEM) imaging at Lawrence Livermore National Lab. Because TEM only provides local void and defect information, ultra small angle x-ray scattering (USAXS) was used to analyze the defects or voids in the coating on a larger scale. The USAXS experiments were carried out at Sector 33 of APS (Chicago, IL, USA), with the beam energy of $12 \mathrm{keV}$. The scattering factor $\mathrm{q}$ is given by $(4 \pi / \lambda) \sin \theta$, where $\lambda$ is the synchrotron wave length and $2 \theta$ the scattering angle. The end station consists of a Bonse-Hart camera, which can measure scattering vectors (q) from about 10-4-100 $\AA-1$. Data were processed using the codes developed for use on this USAXS instrument, and included absolute scattering intensity calibration and slit desmearing. The maximum entropy method, implemented in the "Irena" package for SAS data analysis, was used to determine scatterer size distributions [12]. 
Most studies of film stress in the literature focus on the initial stress development dealing with adatom nucleation and island coalescence. Typical film thickness studied is very thin compared to Be film thickness studied here. Residual stress measurements were carried out insitu on flat Si substrates using a multi-beam optical sensor (MOSS) system manufactured by kSpace Associates, Inc. This system uses an array of parallel laser beams reflecting from the growing substrate surface to measure surface curvature and then convert the measured curvature to stress by using the Stoney formula [11]. The Si substrate has a thickness of $500 \mu \mathrm{m}$ to ensure accurate measurements. The substrate temperature was monitored during MOSS measurements to ensure that the stress measured is mainly due to intrinsic growth stress [11].

\section{RESULTS}

A typical Be coating prepared using magnetron sputtering shows columnar grain structure. The temperature of the substrate during the coating rises to $\sim 150^{\circ} \mathrm{C}$. Since Be melting temperature is $1287^{\circ} \mathrm{C}$, according to Thornton zone model [13], the coating structure is consistent with a transition zone microstructure. At this zone, the adatom does not have enough mobility on the surface for long-range migration and the shadowing effect leads to columnar structure. High resolution TEM pictures show some voids of 100-200 nm diameters along the grain boundaries. These voids are acceptable in size for NIF targets. However, coatings prepared in this way leak $\mathrm{D}_{2}$ fuel gas.

The studies of leak rates at different temperatures indicate that the leak is consistent with molecular flow mechanism and possibly through nanochannels. Detailed TEM images at high resolution reveal that there exist intra-granular channels. Fig. 1(a) shows a TEM image with striking features of intra-granular striation lines. These striation lines are oriented along the growth direction and showed a width of a few nanometers. They are believed to lead to the leakage of $\mathrm{D}_{2}$ gas. As suggested in the literatures these channels are the results of low surface adatom mobility and shadowing effects. Since IPVD can enhance the adatom mobility by supplying more energy to the growing film, it has been used to improve the microstructure and produce leak free Be coatings. Fig. 1(b) shows a high resolution TEM image of Be coating produced by IPVD method. The biasing on the substrate in this case is $-80 \mathrm{~V}$. It is clear from the picture that the striation line defects observed in Fig. 1(a) disappeared. This result is consistent with leak testing results that IPVD produced Be films can hold the $\mathrm{D}_{2}$ gas although some voids can still be seen in the coating along the grain boundaries.

Although TEM characterization reveals the microscopic defects, it is limited to very local scale. USAXS measurements can provide information on defects size and distribution on a larger scale. By measuring the $\mathrm{x}$-ray scattering intensity at different scattering vector $\mathrm{q}$, the scatter size and distribution are measured. The typical magnetron sputtered Be shells showed a void fraction of $5 \%-7 \%$ of the coatingby USAXS measurement, which is consistent with previous measurements of Be coating density of $95 \pm 2 \%$ by weighing [3]. 


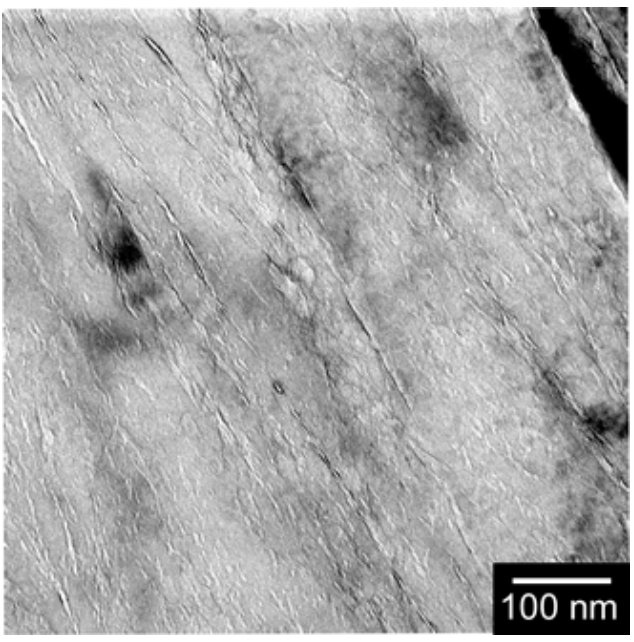

(a)

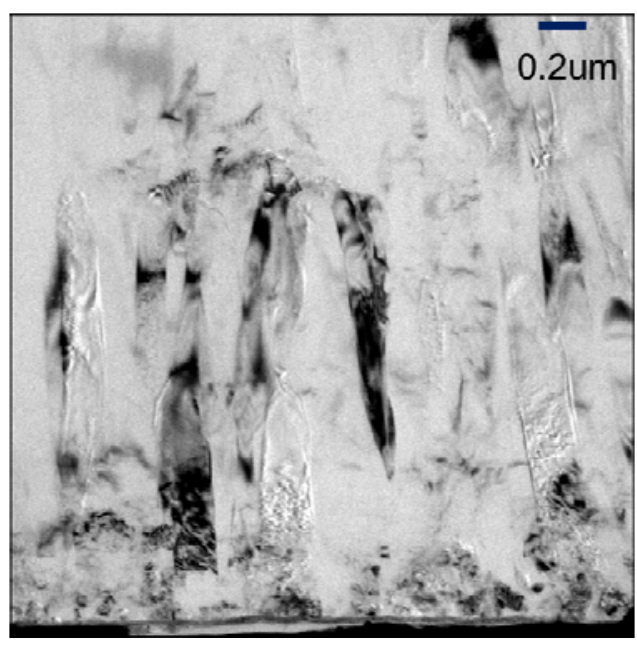

(b)

Fig. 1. High resolution TEM images of regular magnetron sputtering coated Be film showing striation lines of defects (a). An IPVD produced Be coating showed no striation line defects (b).

A comparison of regular magnetron sputtered Be coating with IPVD coating indicated reduction of void size and volume in the IPVD coatings. Figure 2 shows a comparison of two different Be capsules. The "good capsule" refers to an IPVD produced capsule and is leak-free. The "bad capsule" refers to a regular magnetron sputtering produced capsule, which leaks. A rolled Be sheet produced by Brush Wellman was also measured as a reference. It shows that the IPVD-prepared Be capsule scatter intensity is significantly reduced compared to the regular magnetron sputtered Be capsule. The IPVD Be capsule scattering curve is very close to Brush Wellman Be sheet in terms of void scattering intensity, suggesting similar void fraction and sizes. The lower void fraction and size by IPVD compared to regular magnetron sputtering is also consistent with above TEM observations.

In-situ stress measurements using a MOSS system was carried out on flat Si substrates during the coating to gain insight into the stress evolution with thickness. The MOSS measurements of multiple laser beam reflection spot spacing are converted to substrate curvature and then stress. The stress curves at 2 mTorr, 5 mTorr and 10 mTorr for Be coating by magnetron sputtering showed that they initially dipped into compressive stress and then rose to tensile stress. The stress at 2 mTorr Ar pressure sputtering showed more compressive stress. The instantaneous stress shows that after initially rise to tensile, the stress at 2 mTorr, 5 mTorr and 10 mTorr are reaching a plateau indicating new coating does not cause further increasing of the stress. Stress recorded up to $18 \mu \mathrm{m}$ showed the same saturation behavior. 


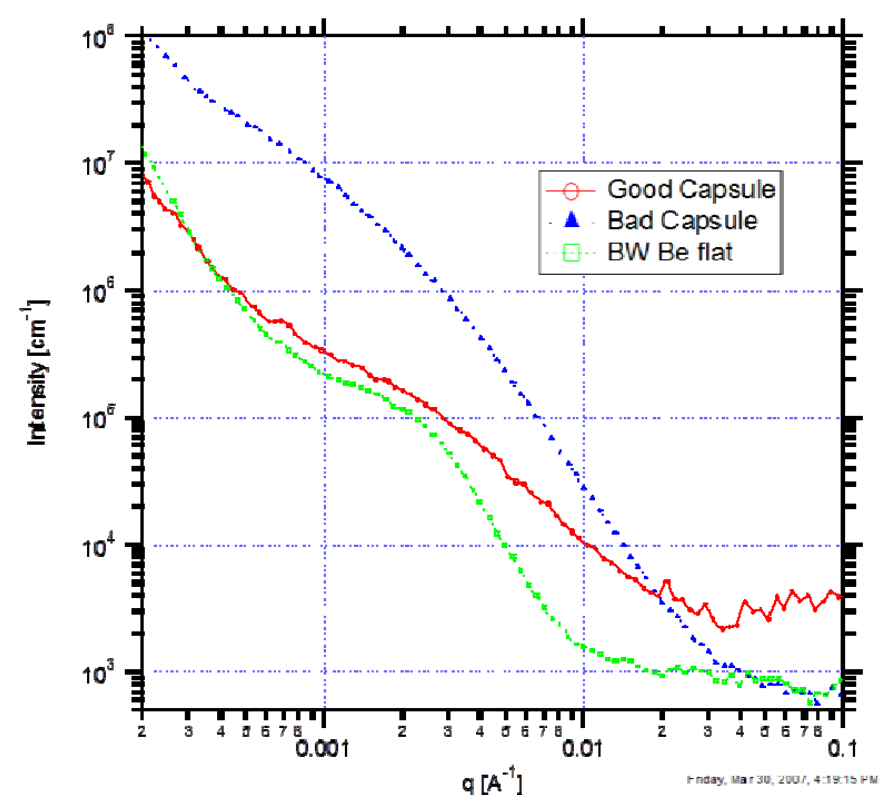

Fig. 2. A comparison of "good capsule" produced by IPVD method and being leak-free, a "bad capsule" produced by regular magnetron sputtering and leaked. A third sample is a flat from Brush Wellman for reference.

IPVD Be coating stress was also measured by in-situ MOSS. As shown in Fig. 3, IPVD at $-20 \mathrm{~V}$ and $-40 \mathrm{~V}$ both showed more compressive stress than regular magnetron sputtering when they were measured at $5 \mathrm{mTorr}$ Ar pressure. The instantaneous stress curves showed that the stress at $-20 \mathrm{~V}$ and $-40 \mathrm{~V}$ biasing approached a plateau and settled at lower stress level than regular magnetron sputtering. The compressive stress under IPVD has been discussed in Detor, et al. paper [11] and was attributed to ion peening effects. The ions with sufficient energy can displace the coating surface atoms and induce atomic rearrangement and compressive stress in the growing film.

\section{CONCLUSION}

Thick Be coatings have been produced by magnetron sputtering. Although the void size and fraction meets the NIF target requirements, Be coatings leaked $\mathrm{D}_{2}$ fuel gas. The IPVD magnetron sputtering was used to improve the coating microstructures and reduced void fraction has been observed by TEM and USAXS measurements. The coating produced by IPVD magnetron sputtering is leak free. The stress of the coating by in-situ MOSS measurements showed stress saturation at larger thickness. The IPVD magnetron sputtered coatings are more compressive although they exhibit the same saturation behavior as regular magnetron sputtering. 


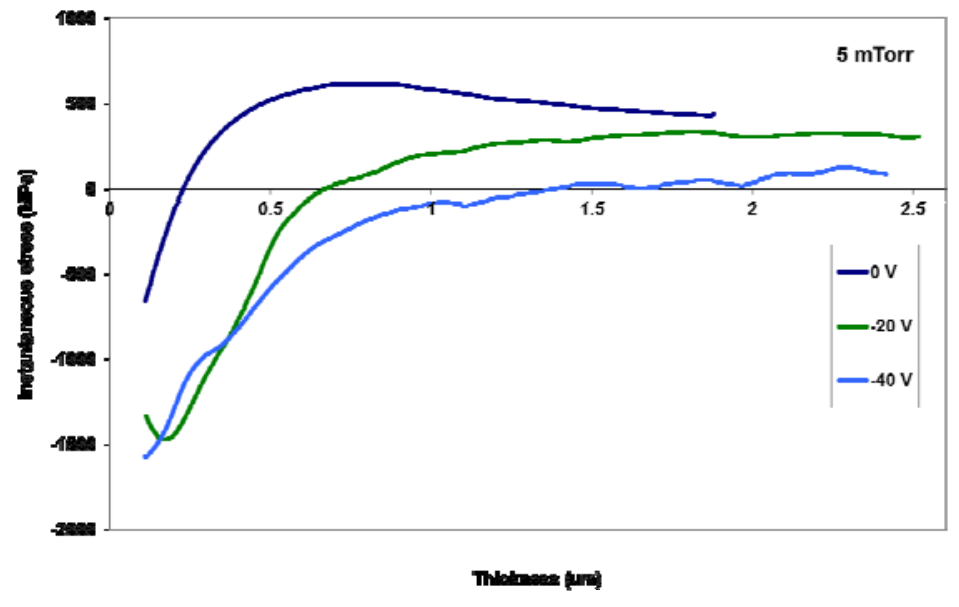

Fig. 3 Instantaneous IPVD Be film stress curves measured by in-situ MOSS. The IPVD coating under $-20 \mathrm{~V}$ and $-40 \mathrm{~V}$ biasing showed more compressive stress than regular magnetron sputtering.

\section{ACKNOWLEDGEMENT}

Work supported by U.S. DOE under contract DE-AC52-06NA27279. Authors would acknowledge J Ilavsky for the USAXS experiments and the APS which was supported by the U.S. Department of Energy under Contract DE-AC02-06CH11357.

\section{REFERENCES}

1. S.W. Haan, D.A. Callahan, M.J. Edwards, B.A. Hammel, D.D. Ho, O.S. Jones, J.D. Lindl, B.J. Macgowan, M.M. Marinak, D.H. Munro, S.M. Pollaine, J.D. Salmonson, B.K. Spears, and L.J. Suter, Fusion Sci. Technol. 55, 227 (2009)

2. R. Mceachern, C. Alford, R. Cook, D. Makowcki, and R. Wallace, , Fusion Technol. 31, 435 (1997)

3. H.W. Xu, A. Nikroo, J.R. Wall, R. Doerner, M. Baldwin, and J.H. Yu, Fusion Sci. Technol. 49, 778 (2006)

4. H.W. Xu, C.S. Alford, J.C. Cooley, L.A. Dixon, R.E. Hackenberg, S.A. Letts, K.A. Moreno, A. Nikroo, J.R. Wall, and K.P. Youngblood, Fusion Sci. Technol. 51, 547 (2007)

5. A. Nikroo, H.W. Xu, K.A. Moreno, K.P. Youngblood, J. Cooley, C.S. Alford, S.A. Letts, and R.C. Cook, Fusion Sci. Technol. 51, 553 (1997)

6. U. Helmersson, M. Lattemann, J. Bohlmark, A.P. Ehiasarian, J.T. Gudmudsson, Thin Solid Films $513,1(2006)$

7. K. Tao, D. Mao, and J. Hopwood, J. Appl. Phys. 91, 4040 (2002)

8. J. Hopwood, Phys. Plasmas, 5, 1624 (1998)

9. D.R. Juliano, D.N. Ruzic, M.M.C. Allain and D.B. Hayden, J. Appl. Phys. 91, 605 (2002).

10. V. Arunachalam, S. Rauf, D.G. Coronell, and P.L.G. Ventzek, J. Appl. Phys. 90, 64 (2001).

This work performed under the auspices of the U.S. Department of Energy by Lawrence Livermore National Laboratory under Contract DE-AC52-07NA27344. 
11. A. Detor, A. Hodge, E. Chason. Y. Wang, H. Xu, M. Conyers, A. Nikroo and A. Hamza, Acta materialia 57, 2055 (2009).

12. J. Ilavsky and P. R. Jemian, J. Appl. Cryst. (2009). 42, 347-353

13. J. A. Thornton, Annu. Rev. Mater. Sci. 7, 239 (1977). 